\title{
OVARIAN CONSERVATION IN PREMENOPAUSAL WOMEN WITH EARLY STAGE ENDOMETRIAL CANCER
}

\author{
O. Kaabia, Z. Rym, G. Nourallah, L. Aymen, S. Aymen, B. Mohamed, K. Hédi. \\ Université de Sousse- Faculté de Médecine de Sousse- Hopital Farhat Hached- \\ LR12ES03- 4000- Sousse- Tunisia-,
}

\section{Introduction :}

The endometrial cancer is a relatively rare condition in premenopausal women, especially before 50 years old. The main treatment for this cancer is surgery.

This study aims to compare the overall survival (OS) and disease free survival (DFS) in young women with earlystage endometrial cancer undergoing hysterectomy with and without ovarian conservation.

\section{Methods :}

We conducted a retrospective study on a prospective monocentric database. We identified all premenopausal women under the age of 50 with stage IA endometrioid adenocarcinoma of the endometrium Grade I who underwent hysterectomy from 2008 to 2018 in our institution, Departement of Gynecology and Obstetrics, Farhat Hached Teaching Hospital. Patients were divided into two groups based on whether they underwent oophorectomy or had ovarian conservation, in addition to radical hystetrectomy.

\section{Results:}

The cohort of 20 women included 5 patients (25\%) who had ovarian conservation and 15 (75\%) who underwent oophorectomy. (Figure 1) .

The rate of ovarian conservation was lower during the last decade.

The OS wax

There were no statistical difference between the ovarian conservation group and the oophorectomy group in OS and DFS, in a Kaplan- Meier analysis, (respectively, $\mathrm{P}=0.073$ and $P=0.200$ ).

\section{Discussion:}

The study shows that in case of a stage IA endometrioid adenocarcinoma of the endometrium grade I, ovarian conservation can be a safe option for women who are premenopausal. However, this study has some limitations : the sample size was relatively small and we did not evaluate the impact of the oophorectomy on the cardiovascular prognosis of the patients. The limited sample size is essentially related to the rarety of this disease in premenopausal women. This can be perfected by created national and international registries for this rare condition for a better understanding in order to offer the patients the best care possible.

\section{Conclusion:}

Ovarian conservation does not adversely affect survival for women with early-stage endometrial cancer .

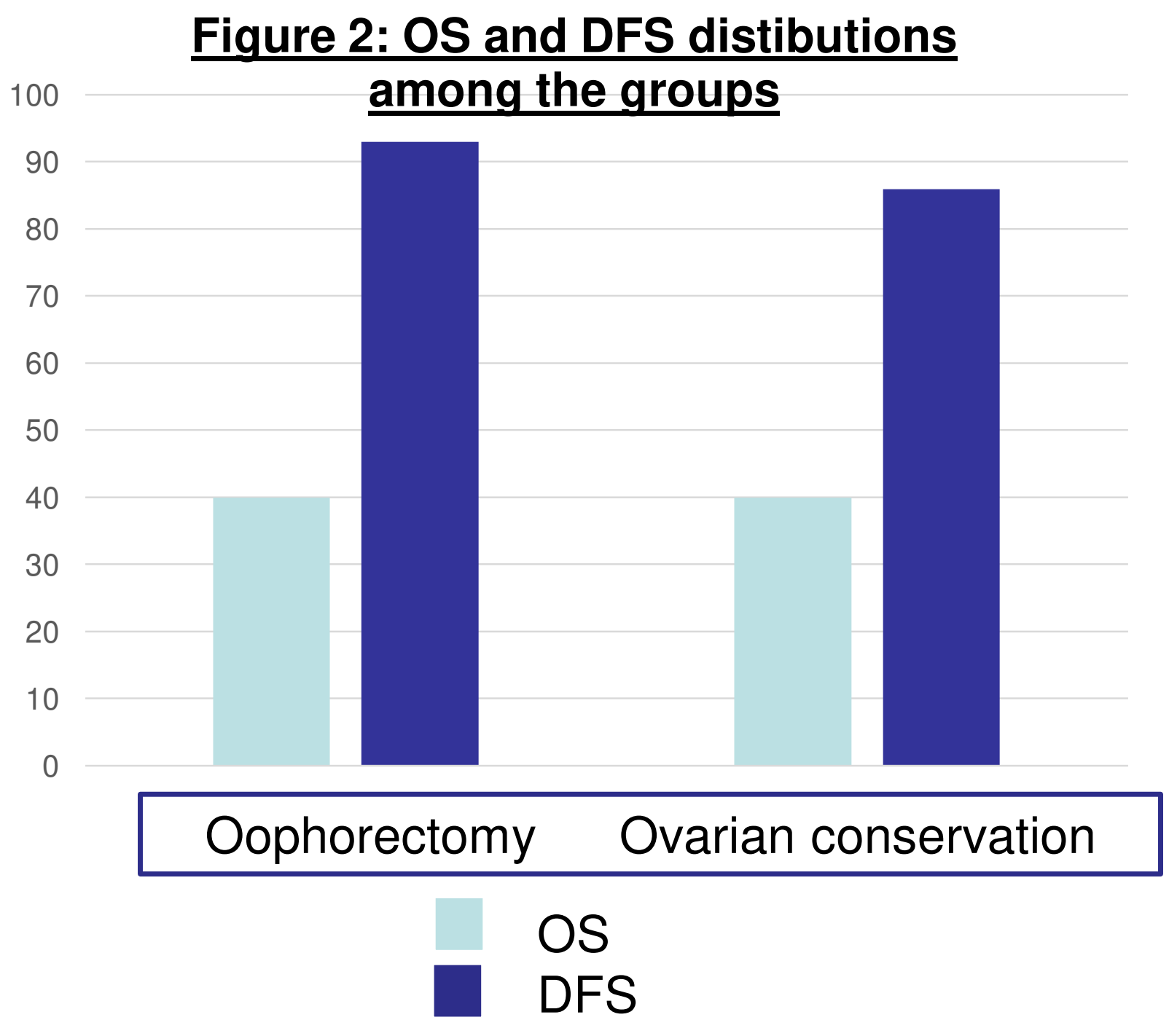

5 ovarian conservation

\section{0 patient s}

\section{No history of infertility}

Figure 1:

12 infertility The chart of the study population

3 no infertility 